\title{
Rehabilitation nutrition care for elderly people with sarcopenia and nutritional disorders
}

\begin{abstract}
Complementary and Alternative Medicine (CAM) and Integrative Medicine (IM) have the characteristic aspect of patient-oriented medicine. Recent problems include elderly people with sarcopenia, frailty, nutritional disorder and daily rehabilitation. It has been evaluated by International Classification of Functioning, Disability and Health (ICF). A circulating process for rehabilitation nutrition care has five stages, which are Assessment/diagnostic reasoning, Diagnosis, Goal setting, Intervention, Monitoring. For goal setting, check list for SMART (Specific, Measurable, Achievable, Relevant, Time) is useful. Diagnosis of rehabilitation nutrition includes the presence or absence of malnutrition, sarcopenia, and deficiency or excess of nutrient intake. When a subject has sarcopenia, we had to check whether it should be improved and whether it can be improved. To prevent iatrogenic sarcopenia will bring the benefit preventing sarcopenia in the whole body, swallowing- and respiratory-related muscles.
\end{abstract}

Keywords: rehabilitation nutrition care, Sarcopenia, nutritional disorder, international classification of functioning, disability and health, ICF, SMART, IMJ
Volume I 3 Issue 3 - 2020

\author{
Akito Moriyasu, ${ }^{1,2}$ Mitsuru Murakami, ${ }^{3}$ \\ Hiroshi Bando 4,5 \\ 'Rehabilitation Research Group for body and heart in Shikoku, \\ Japan \\ ${ }^{2}$ Akiboshi Bright Star training rehabilitation center, Japan \\ ${ }_{3}^{3}$ Japan Masters Athletics, Japan \\ ${ }^{4}$ Tokushima University, Japan \\ ${ }^{5}$ Japan Masters Athletics, Tokushima division, Japan
}

Correspondence: Hiroshi Bando, Tokushima University / Medical Research, Nakashowa I-6I, Tokushima 770-0943, Japan, Tel +8I-90-3 I87-2485 Email pianomd@bronze.ocn.ne.jp

Received: May 26, 2020 | Published: June 16, 2020

\section{Opinion}

In recent years, Complementary and Alternative Medicine (CAM), Integrative Medicine (IM) and Family Medicine (FM) have been in focus worldwide. ${ }^{1}$ Characteristic aspect of CAM, IM and FM would be the patient-oriented medicine from bio-psycho-social points of view. ${ }^{2,3}$ Authors and collaborators have continued several activities of IM as the director of the Shikoku Island division of Integrative Medicine Japan (IMJ). ${ }^{3,4}$ Among these situations, we came to know the necessity of the problems concerning elderly people with sarcopenia, frailty, nutritional disorder and daily rehabilitation. ${ }^{5,6}$ In this article, recent topics would be described on the management of the rehabilitation nutrition care.

The definition of the rehabilitation nutrition has been as follows: ${ }^{6}$ i) it evaluates holistically by the criteria of ICF (International Classification of Functioning, Disability and Health) and also by the situation of sarcopenia, nutritional disorders, deficiency or excess of nutrient intake, ii) it can conduct the diagnosis and goal setting of rehabilitation nutrition, iii) it may induce the optimal content of body functions, quality of life (QOL), activities and participations by adequate methods. They include improving sarcopenia, frailty and nutritional status in patients with frailty and people with a disability by two concepts, which are "nutrition care management in consideration of rehabilitation" and also "rehabilitation in consideration of nutrition". ${ }^{7}$ Consequently, high quality of rehabilitation nutrition should be provided by adequate methods of rehabilitation nutrition care.

There is a circulating process for rehabilitation nutrition care ${ }^{6}$ It has five stages in the following: i) Assessment and diagnostic reasoning; it includes some situations such as malnutrition, frailty, nutritional assessment, ICF guideline. ii) Diagnosis; it has some examples including sarcopenia, cachexia, nutrient intake and nutritional status. iii) Goal setting; we can set adequate goal for nutrition care, using a check list for SMART (Specific, Measurable, Achievable, Relevant, Time-bound). iv) Intervention; medical staffs can provide specific activities for rehabilitation nutrition care, which includes planning, implementation, nutrition care management in consideration of rehabilitation, rehabilitation in consideration of nutrition and so on, v) Monitoring; various patients can be evaluated and monitored from some points of views such as evaluation, body functions, activities, participations, QOL and others. ${ }^{8}$ These five stages are shown in the Figure 1.

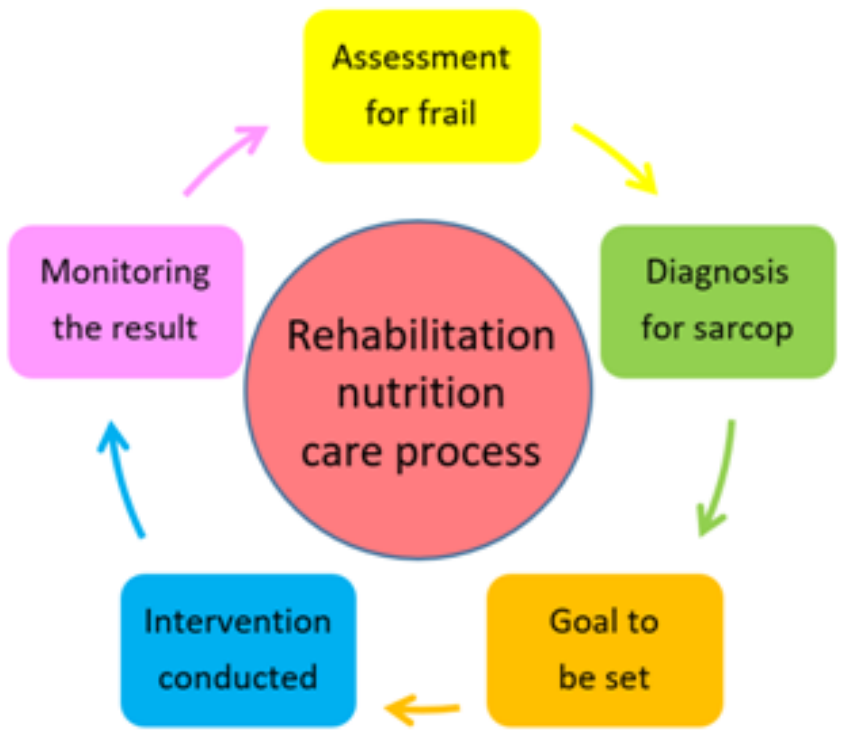

Figure I Five stages of rehabilitation nutrition care process.

From mentioned above, the diagnosis of rehabilitation nutrition includes the investigation of three aspects. They are i) presence or absence of malnutrition, ii) sarcopenia status, and iii) deficiency or excess of nutrient intake. ${ }^{9}$ Consequently, the cause of the impaired condition would be investigated and clarified. As to the setting the goal of the therapy, two important perspectives (A and B) are necessary as follows. 
Regarding A), should the undernutrition and sarcopenia be improved? It must be examined by various medical staffs whether rehabilitation nutrition can be expected to improve swallowing function and ADL. ${ }^{10}$ For example, in patients with complete quadriplegia due to cervical spinal cord injury, improved nutrition cannot be expected to improve the life function. There may be the increased risk or burden of nursing care due to weight gain due to excess fat. Regarding B), can the undernutrition and sarcopenia be improved? In the case of malnutrition and sarcopenia, it is presumed and judged whether the impaired nutritional status can be improved or maintained or whether the deterioration can be prevented depending on its cause and degree. ${ }^{11}$ When we recognize that nutrition can be improved in the case of mild hunger, mild invasion (CRP 3mg/ $\mathrm{dL}$ or less) and pre-cachexia, the adequate goal can be set from the viewpoint of SMART. On the contrary, for severe hunger, refeeding syndrome, severe invasion (CRP $10 \mathrm{mg} / \mathrm{dL}$ or more), refractory cachexia (end stage), the goal cannot be adequately set because of probable difficulty to improve nutritional status. ${ }^{12}$

There have been some actual reports on nutrition and sarcopenia issues in the elderly. Impaired nutrition is common in hospitalized elder patients, inducing poor outcome. In-patients with deconditioning showed malnutrition as $91 \% .^{13}$ Elder patients with anemia and low nutritional index showed poor achievement for rehabilitation. ${ }^{14}$ In standard general medicine ward, $51 \%$ of elderly revealed malnutrition by mini nutritional short-form assessment, and they tended to have higher death and complication rates. ${ }^{15}$ For patients with acute problems, not only current medical treatment but also chronic fundamental insufficient nutrition care would be investigated. Consequently, the necessity of rehabilitation nutrition has been emphasized, including elder patients with physical and swallowing problems. ${ }^{16}$

In this way, rehabilitation nutrition includes wider concepts and actual medical treatments. ${ }^{17}$ They include physically systemic sarcopenia, dysphagia of sarcopenia in swallowing-related muscles, and respiratory-related muscle sarcopenia. ${ }^{18}$ These assessments and treatments usually begin after hospitalization. ${ }^{10}$ However, it is rather ideal that management of taking several measures for sarcopenia before hospitalization may be more beneficial for preventing various sarcopenia. ${ }^{19}$ In our experience, rich-protein foods are often advised to take for subjects with insufficient nutrition condition (Table 1).

Table I Useful foods with rich protein

\begin{tabular}{llll}
\hline & Amount $(\mathrm{g})$ & Protein $(\mathrm{g})$ & Carbohydrate \\
\hline Egg & $60 \mathrm{~g}$ & 7.4 & 0.2 \\
Cheese & $20 \mathrm{~g}$ & 3.7 & 0.2 \\
Roast Ham & $20 \mathrm{~g}$ & 3.8 & 0.4 \\
Milk & $100 \mathrm{~g}$ & 3.4 & 4.9 \\
Green Beans & $120 \mathrm{~g}$ & 2.0 & 6.0 \\
\hline
\end{tabular}

Medical personnel should note that there is "iatrogenic sarcopenia" in actual medical practice. ${ }^{7}$ There are i) sarcopenia due to improper resting and abstinence in the ward, ii) nutrition sarcopenia due to improper nutritional management in the hospitals, and iii) sarcopenia due to iatrogenic diseases from medical reason. These are likely to occur mainly in acute care hospitals. One example is shown as a patient with aspiration pneumonia. In such case, standard instruction in early stage would be to rest and keep on the bed, to prohibit eating or drinking, and to give infusion of water and electrolyte only. ${ }^{6}$
To prevent iatrogenic sarcopenia can lead to the better situation preventing sarcopenia in the whole body, swallowing-related and respiratory-related muscles. It also leads to the prevention of bedridden, eating/swallowing disorders, and respiratory dysfunction. The ideal management is to prevent iatrogenic sarcopenia by conducting an appropriate evaluation and treatments. They include early bed leaving, early oral intake, and proper nutritional management about 2 days after hospitalization. ${ }^{20}$

\section{Conclusion}

The background and situation of the subject are diagnosed. After that, by appropriately performing rehabilitation and nutritional management at the same time, it becomes possible to maintain life function and QOL preventing deterioration. In the case of nutritional disorder, it is effective to start early to improve nutrition, and to provide resistance training if needed. A preferable treatment policy would be desired that includes patient-oriented perspective from CAM and IM points of view.

\section{Acknowledgments}

None.

\section{Conflicts of interest}

Author declares that there are no conflicts of interest.

\section{Funding}

None.

\section{References}

1. Berretta M, Rinaldi L, Taibi R, et al. Physician Attitudes and Perceptions of Complementary and Alternative Medicine (CAM): A Multicentre Italian Study. Front Oncol. 2020;10:594.

2. Hirai Y, Bando H, Yoshioka A, et al. Music and Man in Art: The Future of Media and Technology. Global J Arts Social Sci. 2020;2(1):116.

3. Bando H, Yoshioka A, Nishikiori Y. Various Care Option of Integrative Medicine from the Viewpoint of Patient-Oriented Medicine. Int J Conf Proc. 2020;2(1):000529.

4. Nishikiori Y, Bando H, Yoshioka A, et al. Trials of Additional Effective Movements for Music Therapy Session for the Elderly. Curr Res Complement Altern Med. 2020;4:138.

5. Murakami M, Moriyasu A, Bando H. Better Application of Exercise Prescription with Adequate Supports to All People. Biomed Sci J. 2020;1:4.

6. Wakabayashi H. Rehabilitation nutrition in general and family medicine. J Gen Fam Med. 2017;18(4):153-154.

7. Nagano A, Nishioka S, Wakabayashi H. Rehabilitation Nutrition for Iatrogenic Sarcopenia and Sarcopenic Dysphagia. J Nutr Health Aging. 2019;23(3):256-265.

8. Hansen T, Kjaersgaard A. Item analysis of the Eating Assessment Tool (EAT-10) by the Rasch model: a secondary analysis of cross-sectional survey data obtained among community-dwelling elders. Health Qual Life Outcomes. 2020;18(1):139.

9. Savas S, Yilmaz M. Self Reported Dysphagia is not Associated with Sarcopenia Defined by the Revised EWGSOP2 Criteria and Regional Thresholds at the Hospital Among Ambulatory Older Patients. Mater Sociomed. 2019;31(4):253-257.

10. Dellis S, Papadopoulou S, Krikonis K, et al. Sarcopenic Dysphagia. A Narrative Review. J Frailty Sarcopenia Falls. 2018;3(1):1-7. 
11. Azzolino D, Arosio B, Marzetti E, et al. Nutritional Status as a Mediator of Fatigue and Its Underlying Mechanisms in Older People. Nutrients. 2020;12(2):444.

12. Kagifuku Y, Tohara H, Wakasugi Y, et al. What Factors Affect Changes in Body Composition and Swallowing Function in Patients Hospitalized for Oral Cancer Surgery? Clin Interv Aging. 2020;15:1-7.

13. Wakabayashi H, Sashika H. Association of nutrition status and rehabilitation outcome in the disuse syndrome: a Retrospective Cohort Study. Gen Med. 2011;12:69-74.

14. Goto R, Watanabe H, Tanaka N, et al. Factors associated with recovery of activities of daily living in elderly pneumonia patients. Gen Med. 2015;16:68-75.

15. Sato K. Mini Nutritional Assessment Short-Form (MNA-SF) predicts clinical outcomes: cohort study of small-sized hospital in Japan. J Gen Fam Med. 2016;17(1):90-98.
16. Momosaki R. Rehabilitative management for aspiration pneumonia in elderly patients. J Gen Fam Med. 2017;18(1):12-15.

17. Azzolino D, Damanti S, Bertagnoli L, et al. Sarcopenia and swallowing disorders in older people. Aging Clin Exp Res. 2019;31(6):799-805.

18. Okazaki T, Ebihara S, Mori T, et al. Association between sarcopenia and pneumonia in older people. Geriatr Gerontol Int. 2020;20(1):7-13.

19. Azzolino D, Passarelli PC, De Angelis P, et al. Poor Oral Health as a Determinant of Malnutrition and Sarcopenia. Nutrients. 2019;11(12):2898.

20. Fujishima I, Fujiu-Kurachi M, Arai H, et al. Sarcopenia and dysphagia: Position paper by four professional organizations. Geriatr Gerontol Int. 2019;19(2):91-97. 